\title{
Let Doctors Be Doctors -Don't Let Engineers Determine Injuries
}

\author{
John J Smith* \\ Licensed Professional Engineer, Missouri, USA
}

Received: August 23, 2017; Published: September 13, 2017

*Corresponding author: John J Smith, PE, MSS, BSGP, Licensed Professional Engineer, Missouri, USA, Tel: 303-840-0549; Email: JJSakaJTM@aol.com

\section{Don't Let Engineers Determine Injuries}

A scenario plays out every day in America. A collision occurs, the party is transported by EMS, or sees the Doctor soon afterwards, and a chain of medical treatment ensues. The medical providers report objective findings and opine that the treatments are necessary and reasonable. Despite this, when reimbursement is sought, a report appears from an engineer/scientist and without scientific or engineering basis the author claims that the injury is not related to the event. To support the opinions of the hired analyst, a series of assertions are made in an attempt to discredit the treating providers' opinions. While not predicated upon any valid engineering or science, the medical providers may have trouble being reimbursed for the reasonable and necessary services.

This article looks at many of the common misconceptions regarding traumatic events and provides the reader with the knowledge necessary to address the inaccurate claims. In the field of biomechanics, the key issue is; were there forces, loads and moments applied in the areas where the medical providers have found the injuries. Engineers should not be used to discount medical treatments, let Doctors be Doctors.

Areas that will be addressed are
A. The claim of an injury threshold.
B. The comparison of a traumatic event to daily activities.
C. The damage to a vehicle determines if a person is injured.
D. Safety optimized volunteer testing directly correlated to non-safety optimized real world events.
E. Change in velocity is important in a motor vehicle collision.

Abbreviations: NHTSA: National Highway Traffic Safety Administration

\section{Injury Threshold Claims}

There are often discussions of limits of exposure in the epidemiological community and disease vectors are commonly studied. However, from a biomechanical standpoint the issue of threshold and injury are less clear. An injury threshold can be described as a level of damage or force above which an injury will occur. However, failure of biological material is not linear and often not repeatable. As an example, SAE J885 discusses the failure of a femur under loading and notes femur fractures from forces as little as $4,230 \mathrm{~N}$ while other trials have femurs withstanding a force of $17,130 \mathrm{~N}$ with no damage. 20 trials were run with different femurs and there was no apparent baseline force found for the point at which a femur might fracture. The National Highway Traffic Safety Administration (NHTSA) provides performance standards for vehicles, not threshold criteria.

Despite these issues, a threshold has been proposed for motor vehicle collisions based on grossly dissimilar testing of potentially biased volunteers. The most common approach seen in motor vehicle collisions is a claim that below a $5 \mathrm{mph}$ change in velocity injuries are not expected. Many injured parties may have their injuries dismissed while needing treatment due to this arbitrary threshold. To understand this unsupported claim, it is helpful to discuss the origins of the theory. In the early 1990s, a group of consultants who testify primarily for insurance companies and automobile manufacturers decided to run a set of safety optimized kinematic studies, using themselves as volunteers. Only individuals with a financial interest in the company were allowed to participate in order to limit the potential liability. Steps were specifically taken to prevent injury such as not impacting the researcher by surprise, the use of bite blocks and other safety measures.

The consultants reported their observations regarding their own injuries and then asserted that since they were not injured, they had determined a "threshold" for injury. It has not been established that these researchers did not have long term complications but regardless of this, it was asserted that the threshold for injury was a 4 to $5 \mathrm{mph}$ change in velocity [1]. After the initial work of the consultants, several other groups of consultants who worked 
primarily for insurance companies and manufacturers ran tests using themselves as subjects. The fallacy of researchers using themselves as subjects was demonstrated by the assertion of the manager of one company who stated when discussing their paper that the researchers were hired to prove an injury would not occur. However, even though this paper clearly cannot be used to establish an injury threshold, it is routinely cited as an example of a case where an older woman with pre-existing radiographic findings was not injured. Omitted from the discussion is the fact that the woman had a financial interest in not being injured and violated standard research protocol.

Since that time, it has become routine for an accident reconstructionist to assert that the change in velocity of a collision was $5 \mathrm{mph}$ or less, regardless of the damage to the vehicles. This occurs despite the fact that the researchers failed to establish an actual threshold for the public at large. The researchers did not even establish that change in velocity is the relevant measure of injury potential in rear impacts. As an example, the differential movement of L3 versus L4 versus L5 is more important than the actual change in velocity or the peak acceleration. Subsequently, some researchers have attempted to test non-employees, although still in safety optimized conditions. This research has shown that symptoms can occur in collisions with a change in velocity of only $2.5 \mathrm{mph}$ and unpublished results reported symptoms with a change in velocity of only $1 \mathrm{mph}$.

Missing in this approach is a consideration of clearly identified biomechanically relevant aggravating factors which can increase the rate of injury [1]. Also absent is the discussion of injury studies that have looked at actual collisions of the motoring population versus using preselected, safety optimized, volunteers. As discussed below, studies of actual people refute the claim of an identified injury threshold.

\section{Injury Rates in Rear Impacts}

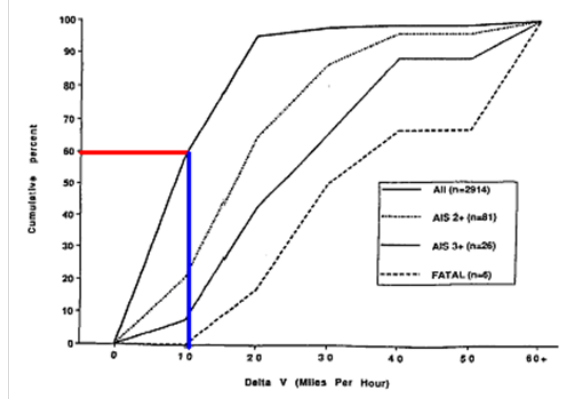

Figure 1: NASS Injury Rates in Rear Impacts, with permission, Murray Mackay.

It has never been established that there is a minimum speed change value below which people are not injured in real collisions. To the contrary, Professor Murray Mackay has analyzed more than 2914 actual accidents reported in the U.S. National Accident Sampling System and showed that there is no threshold speed change value for injury in real life (as opposed to staged) collisions [1] (Figure 1). While Mackay concentrated on rear impacts,
Kullgren and Kraft support the lack of injury threshold in rear impacts and demonstrated that there is also no injury threshold in frontal impacts [2]. Numerous other resources also support the lack of a threshold [3-9]. Biomechanically, the reason for this is obvious. It has never been established that change in velocity is the relevant factor. The often stated injury threshold is not based on an accepted methodology and is refuted by readily available data. Many proponents of the threshold argument fail to incorporate any of the many injury studies that are available that would establish that there is not an injury threshold. For example, paper showed injuries at below the "threshold" [10].

\section{Traumatic Events Compared to Daily Activities}

Traumatic motor vehicle collisions are often improperly compared to daily activities in an attempt to set a model for the scale and types of injuries that may occur in said collision. This comparison is fundamentally flawed for many reasons. These include the definition of force, the definition of a resultant, learned response, internally generated forces, load rates and other issues. Definition of Force: A force is a vector and by definition a vector has both a size and a direction [1]. For two forces to be the same, they must be identical in direction, size and duration. The force must also be applied in the same location. If a researcher wished to show that a traumatic motor vehicle collision is equivalent to a daily activity, it would be necessary to show that for both cases all forces are the same in the location applied, duration, sequence and initial position of the person. This assumes that the test subjects are identical to the crash victims in terms of age, gender, height, weight, resilience, constitution, and predisposition to injury. There is not enough test data available to be able to reliably compare the vectors involved in daily activities to data gathered from motor vehicle collision testing. However, there is sufficient data to prove the comparison is invalid.

Addressing each of the issues individually reveals the inherent fallacies in the approach.

a. Gs are not vectors and are therefore not forces. At a basic level, this is clear since Newton's Second Law is Force is equal to mass times acceleration. Since Gs are acceleration, there is no mass and therefore no force. This has created an imprecise term "G-Force". While popular in discussing fighter pilots and astronauts, it has no applicability to most motor vehicle collisions.

b. Peak acceleration does not describe the net effect on the occupant. First the peak acceleration on the vehicle is not the peak acceleration on the person and fails to capture the complexity of a motor vehicle collision. (Figure 2) is data from the NHTSA for a rear impact on a Chevrolet S-10. It is clear that a single value would not capture the complexity of the collision.

c. Peak acceleration on the person is not repeatable. (Figure 3) is data from volunteer testing. Even under laboratory conditions when many of the dozens of variables can be controlled, significant variations are possible for a particular speed. Variations exceeding 800 percent for the same velocity under controlled experimentation [11]. Research has also 
demonstrated variations of 100 percent in a single individual tested under similar conditions. It is worth noting that the peak acceleration represents only one of the hundreds of forces the occupant was subjected to.

\begin{tabular}{|c|c|c|c|c|c|c|}
\hline NAME & Unit & $\operatorname{Max}$ & msec & Min & msec & Comment \\
\hline V2P1 Head $x$ & $g$ & 77.6 & 70.2 & -3.6 & 252.6 & \\
\hline V2P1 Head y & $g$ & 5.6 & 68.3 & -3.1 & 154.4 & \\
\hline V2PI Head z & $\mathrm{g}$ & 28.3 & 69.7 & -11.2 & 76.9 & \\
\hline V2P1 Head Resultant & $\mathrm{g}$ & 82.5 & 70.2 & 0.0 & -20.9 & \\
\hline V2PI Head Y Displacement & $\mathrm{deg}$ & 8.0 & 68.3 & -27.4 & 364.5 & $\mathrm{f} 2=0.287 \mathrm{~Hz} ; \mathrm{f} 4=0.001 \mathrm{~Hz}$ \\
\hline V2P1 Upper Neck Fx & $\mathrm{N}$ & 437.4 & 72.4 & -117.6 & 344.4 & \\
\hline V2P1 Upper Neck Fy & $\mathrm{N}$ & 117.4 & 86.3 & -145.6 & 157.5 & \\
\hline V2P1 Upper Neck Fz & $\mathrm{N}$ & 377.5 & 90.4 & -2661.8 & 75.7 & \\
\hline V2P1 Upper Neck F Resultant & $\mathrm{N}$ & 2672.4 & 75.7 & 0.5 & -21.5 & \\
\hline V2PI Upper Neck Mx & $\mathrm{N}-\mathrm{m}$ & 9.1 & 98.3 & -8.7 & 162.2 & \\
\hline V2P1 Upper Neck My & $\mathrm{N}-\mathrm{m}$ & 79.1 & 73.8 & -18.2 & 116 & \\
\hline V2PI Upper Neek Mz & $\mathrm{N}-\mathrm{m}$ & 2.9 & 95.6 & -4.2 & 146.8 & \\
\hline V2P1 Upper Neck M Resultant & $\mathrm{N}-\mathrm{m}$ & 79.2 & 73.8 & 0.0 & -26.4 & \\
\hline V2PI Lower Neck FX & $\mathrm{N}$ & 727.7 & 72.2 & -312.9 & 112.6 & \\
\hline V2PI Lower Neck Fy & $\mathrm{N}$ & 201.7 & 66.5 & -98.7 & 172 & \\
\hline V2PI Lower Neck Fz & $\mathrm{N}$ & 280.1 & 90.8 & -2668.9 & 75.9 & \\
\hline V2P1 Lower Neck F Resultant & $\mathrm{N}$ & 2735.5 & 75.9 & 0.4 & -94.2 & \\
\hline V2P1 Lower Neck Mx & $\mathrm{N} \cdot \mathrm{m}$ & 20.6 & 90.8 & -33.3 & 162.7 & \\
\hline V2P1 Lower Neck My & $\mathrm{N}-\mathrm{m}$ & 61.3 & 77.6 & -69.6 & 64.8 & \\
\hline V2P1 Lower Neck Mz & $\mathrm{N}-\mathrm{m}$ & 18.1 & 65.0 & -5.4 & 132.7 & \\
\hline V2P1 Lower Neck M Resultant & $\mathrm{N}-\mathrm{m}$ & 72.7 & 64.8 & 0.0 & -56.2 & \\
\hline V2P1 Lower Neck $\mathrm{x}$ & $\mathrm{g}$ & 30.5 & 62.1 & -9.6 & 108.2 & \\
\hline V2P1 Chest $\mathrm{x}$ & $\mathrm{g}$ & 25.9 & 61.8 & -4.2 & 112.1 & \\
\hline V2P1 Chest y & $\mathrm{g}$ & 48.4 & 76.3 & -196.3 & 105.5 & Transducer failed \\
\hline V2P1 Chest z & $\mathrm{g}$ & 13.3 & 75.4 & -6.4 & 121.2 & \\
\hline V2PI Chest Resultant & $\mathrm{g}$ & 196.3 & 105.5 & 0.0 & -73.8 & \\
\hline V2P1 Chest Displacement & $\mathrm{mm}$ & 0.4 & 114.8 & -2.0 & 87.8 & \\
\hline V2P1 Chest Y Displacement & $\operatorname{deg}$ & 0.9 & 56.5 & -15.9 & 390.2 & $f 2=0.296 \mathrm{~Hz} ; f 4=0.001 \mathrm{~Hz}$ \\
\hline V2P1 Pelvic $x$ & $\mathrm{~g}$ & 19.6 & 88.1 & -3.5 & 120 & \\
\hline V2PI Pelvic y & $g$ & 1.7 & 76.1 & -36.3 & 42.9 & Wire cut at $23 \mathrm{~ms}$ \\
\hline V2PI Pelvic z & $\mathrm{g}$ & 14.6 & 77.2 & -5.9 & 119.4 & \\
\hline V2P1 Pelvic Resultant & $\mathrm{g}$ & 36.3 & 42.9 & 0 & -98.8 & \\
\hline V2P1 Left Femur & $\mathrm{N}$ & 604.5 & 82.9 & -974.9 & 124.1 & \\
\hline V2PI Right Femur & $\mathrm{N}$ & 261.7 & 89.4 & -572.1 & 73.5 & \\
\hline V2PI Head to Chest & $\operatorname{deg}$ & 13.8 & 154.1 & -14.3 & 237.8 & \\
\hline V2 Driver Lap Belt & $\mathrm{N}$ & 266.2 & 258.9 & -24.7 & 34.8 & \\
\hline V2 Driver Belt Spoolout & $\mathrm{mm}$ & 324.7 & 78.2 & .528 .1 & 223 & Data invalid after $75 \mathrm{~ms}$ \\
\hline $\mathrm{V} 2 \mathrm{P} 2$ Head $\mathrm{Ax}$ & $\mathrm{g}$ & 113.5 & 78.6 & -5.3 & 170.9 & \\
\hline V2P2 Head Ay & $\mathrm{g}$ & 1.2 & 289.8 & -10.5 & 79 & \\
\hline $\mathrm{V} 2 \mathrm{P} 2 \mathrm{Head} \mathrm{Az}$ & $\mathrm{g}$ & 40.7 & 78.8 & -6.1 & 111.9 & \\
\hline V2P2 Head A Resultant & $\mathrm{g}$ & 120.9 & 78.6 & 0 & -82.1 & \\
\hline V2P2 Head Y Displacement & $\operatorname{deg}$ & 29.6 & 78.6 & -42.5 & 172.8 & $\mathrm{f} 2=0.292 \mathrm{~Hz} ; \mathrm{f} 4=0.001 \mathrm{~Hz}$ \\
\hline V2P2 Upper Neck Fx & $\mathrm{N}$ & 297.9 & 66.4 & -145.9 & 169.1 & \\
\hline V2P2 Upper Neck Fy. & $\mathrm{N}$ & 50.4 & 288.8 & -62.7 & 78.9 & \\
\hline V2P2 Upper Neck Fz & $\mathrm{N}$ & 1311.7 & 78.6 & -208.5 & 108.4 & \\
\hline V2P2 Upper Neck F Resultant & $\mathrm{N}$ & 1317.5 & 78.6 & 0.1 & -93 & \\
\hline
\end{tabular}

Figure 2: NHTSA Test Data.

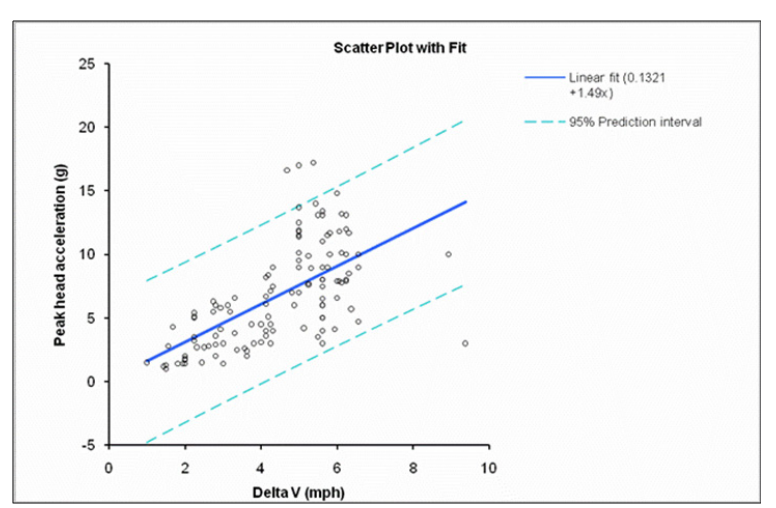

Figure 3: Peak Acceleration Compared to Change in Velocity.

d. The resultant is what matters. If a force is applied at C5 and then at a different time, another force is applied to
C6, the vertebrae were not moved as solid object but rather differentially. This will apply stresses, strains, sheering and torqueing to the disc and associated ligaments, tendons and muscles. Full scale testing of cadavers demonstrates that in a rear impact the cervical column is twisted out of its normal shape into an S-curve. Full scale NHTSA data also demonstrates the differential movement of the upper and lower cervical column (Figure 4). Data from NHTSA test on an Acura RSX (Figures $5 \& 6$ ). As the data clearly demonstrates, the motion of the upper neck is different than the motion of the lower neck.

e. Numerous other factors such as learned response, internally generated forces, load rates and other issues are also important. We know how to walk down stairs; we do not know how to be in a car crash. Daily activities typically have a low load rate whereas motor vehicle collisions are associated with high load rates. The visco elastic nature of biological tissues prevents a direct comparison to normal daily activities. 


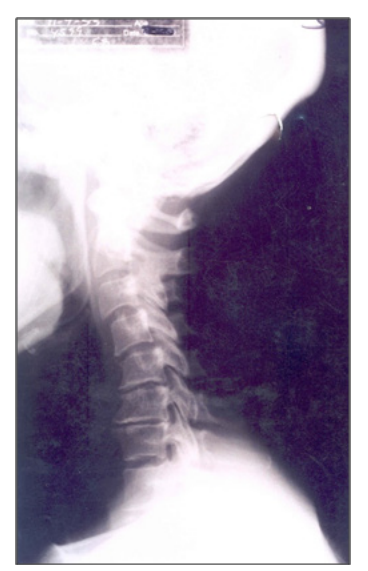

Figure 4: "S" Shape.

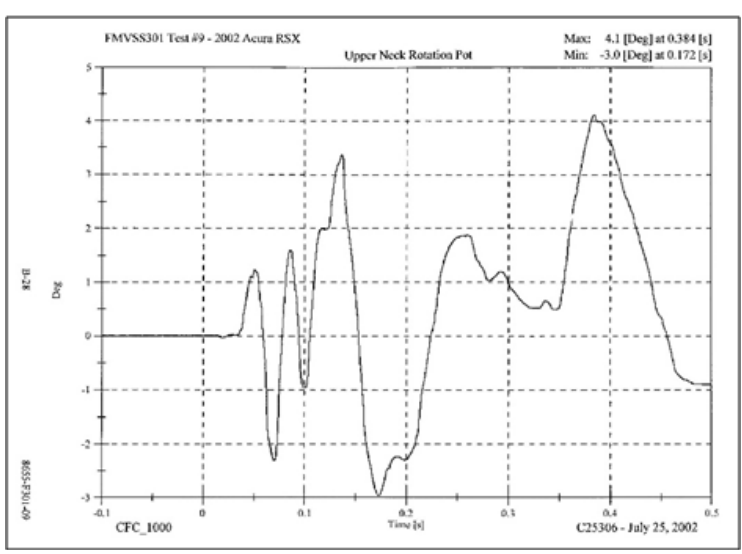

Figure 5: Upper Neck Rotation.

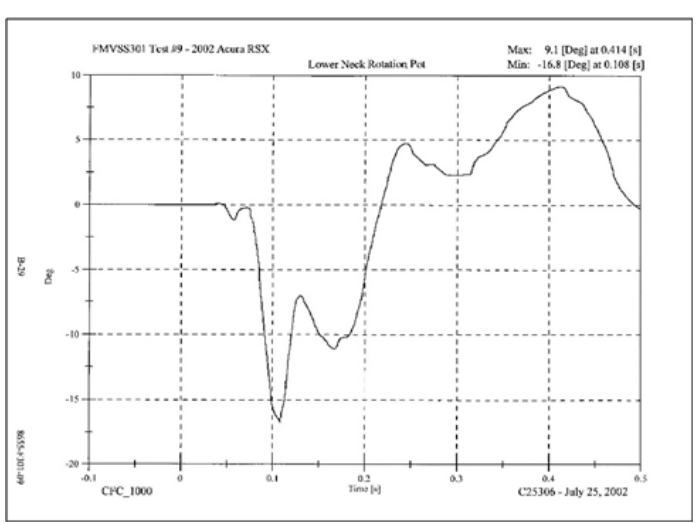

Figure 6: Lower Neck Rotation.

The Damage to the Vehicle Determines if the Person is Injured

A variation on the theme of an injury threshold is the concept of looking at the damage to the vehicle and asserting a person was not injured. While damage can be informative as to the types and locations of forces, it cannot establish a person was not injured. If there is enough energy to bend steel, there is usually sufficient energy to injure people. If there is not enough energy to bend steel, there may still be sufficient energy to injure people. There are times when a vehicle has very little apparent damage yet the driver and passengers may experience very traumatic injuries.

Research using the NASS database demonstrates that it is not possible to look at a vehicle and predict the injury suffered by the occupant [11]. NASS database with confirmed injuries (Figures 7-9). From merely looking at the pictures it would not be possible to know that an occupant in the vehicle (Figure 7) suffered a cervical disc herniation, while an occupant in the vehicle (Figure 8) was not injured and an occupant in the vehicle (Figure 9) was fatally injured. Even if the damage was indicative of the injury, photographs often do not show the full extent of the damage. As an example, modern bumpers need to be disassembled to establish the damage to the reinforcement. Repair estimates often include components that cannot be seen in the images.

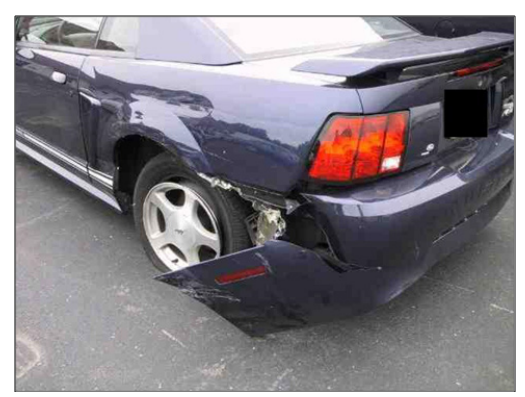

Figure 7: Image from NASS Database.
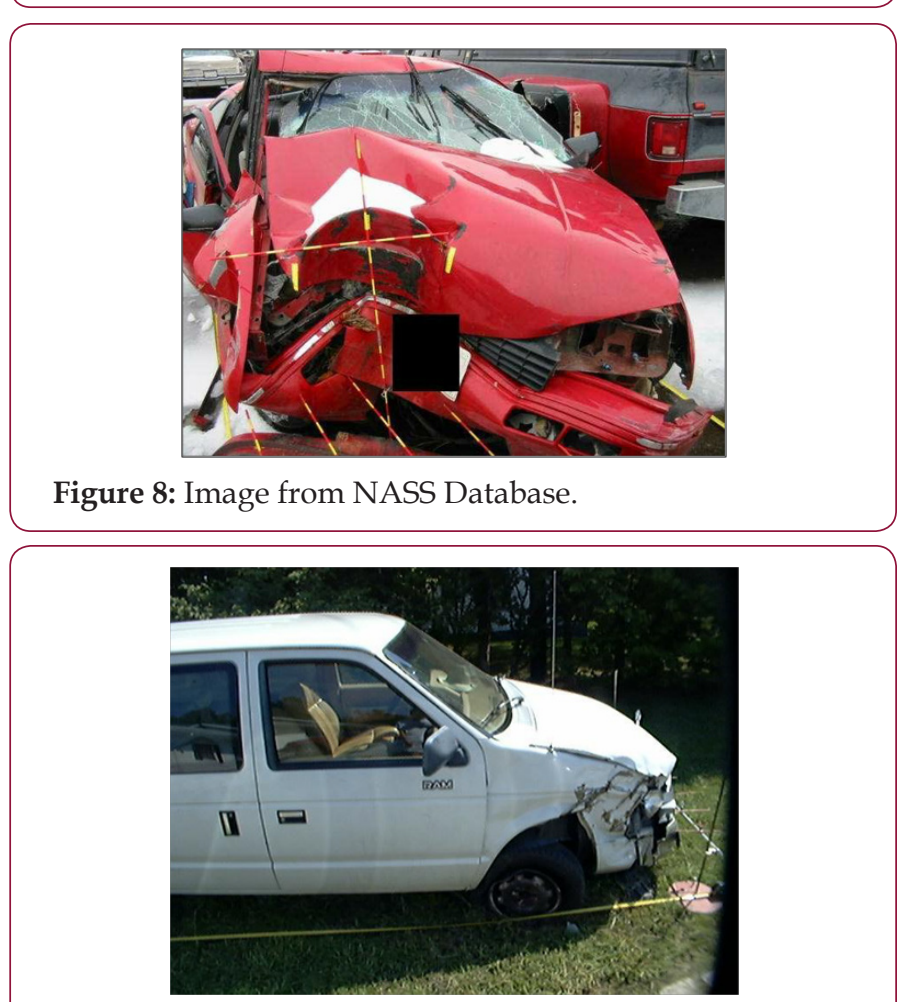

Figure 9: Image from NASS Database.

Safety Optimized Volunteer Testing Directly Correlate to Non Safety Optimized Real World Events

There has been limited testing conducted on vehicular collisions, and the testing that has been done does not represent 
real world conditions well enough to be referenced in litigation. Still these studies are often cited as reliable sources to assert a patient should not have been injured. The limitations of volunteer testing in any form are readily apparent. The injuries to a person in a real collision cannot be equated to injuries sustained by crash test volunteers for numerous reasons. In crash tests, safety is optimized. Often the volunteer is a healthy young male. Sometimes special seat belts are used. Bite blocks are employed to prevent injury to the teeth and jaw. The test subject is aware that the crash is about to take place. The test subject is positioned to receive the forces of the crash. The subject is looking straight ahead and sitting upright. In the few cases where a crash subjects head was turned, the injuries suffered were significantly worse.

The sample size in crash tests is quite small. These tests are fairly expensive, therefore the number of vehicles employed and the number of test subjects used is limited. The statistical accuracy of these tests is questionable considering the number of vehicle speeds that are tested and the various vehicle impact angles that need to be evaluated. This uncertainty is compounded by the variations in the human body's tolerance to injury as a function of age, gender, position in the seat, and possible predisposition to injury as a result of prior medical problems. Even some of the authors of these tests state that they cannot be extrapolated to the general public.

Expounding upon these limitations are the biomechanically relevant aggravating factors. Any biomechanical discussion would be incomplete without a discussion of these aggravating factors. Among the identified factors are; seatbelt usage/tightness, gender, predisposition to injury, surprise, applied angular accelerations, multiple impacts and other variables. It is worth noting that volunteers are almost never tested in collisions that result in damage to the vehicles since the risk of injury is too high. Finally, the number of permutations available in the way a motor vehicle collision can occur effectively eliminates all volunteer tests for asserting an injury could not occur. It is well established that there are individual variances as well as variances in collisions. Assuming that there were only 16 binary variables, the number of permutations is 65,536 . Sixty-five thousand tests have not been run. However, in reality, the quantity of variables is much larger and they are not all binary. The actual number of permutations is easily over a million. Therefore, to take a controlled test and infer an injury did not occur is invalid. It is normally safe to assume that no one has been tested under the conditions a patient was subjected to change in Velocity is Important in a Motor Vehicle Collision. The final topic builds on the other ones discussed. The use of change in velocity to discount injuries is effectively unique to the insurance industry. Since there is no known threshold for injury and no one has ever been tested under the conditions a patient was subjected to, it must be a medical decision as to what injuries a person has sustained. An actual biomechanical analysis builds on the diagnoses supplied by the treating medical providers. Once the injuries have been identified, two questions can be answered. First, were there forces, loads and moments applied where the treating medical providers have found injuries? The second question that can be addressed, are these the types of injuries known to occur in a given impact?

As an example, in a rear impact, the cervical column naturally pivots at C5/C6. If there is a rotational component, the location can move up and down the spine. If a treating medical provider has found an injury in the lower cervical column after a rear impact, there would be no basis to discount this injury or assert it occurred elsewhere. When the NASS investigators perform an analysis of the injuries, it is not done to discount or disprove an injury. It is used to understand how a diagnosed injury occurred. Note the absence of any discussion regarding peak accelerations, G-forces and change in velocity. Figure 10 is from a NASS investigation.

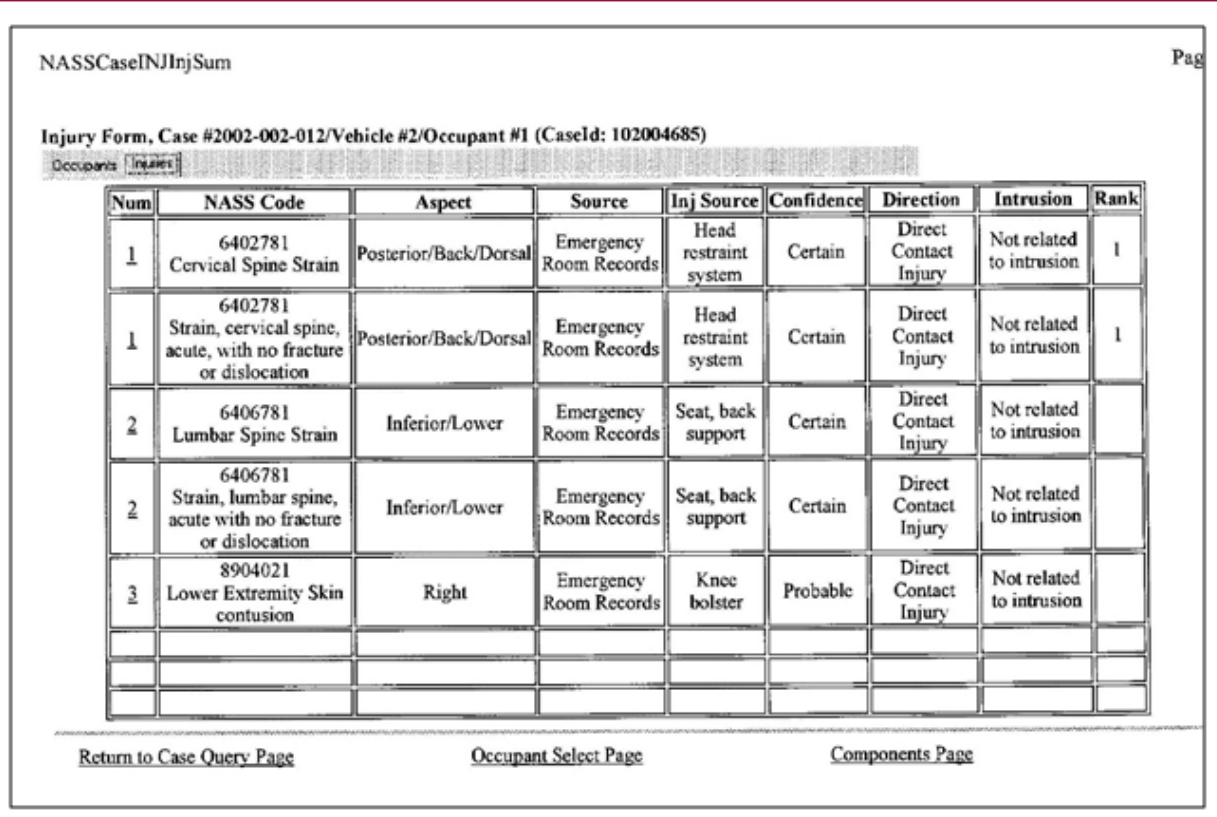

Figure 10: NASS Data. 


\section{Conclusion}

As the data above demonstrates, it is not possible to look at the vehicle damage in most types of collisions and determine if a person has or has not been injured. There is no engineering or scientific basis for asserting a person was not injured in a collision unless there were no forces applied. A valid scientific approach is to start with the diagnosed injuries and determine if forces were applied where the injuries are located. If there is a match in location, the researcher can consider if the injuries reported are consistent with the injuries known NASS Data (Figure 10).

\section{References}

1. Low Speed Rear Impact Collision TOPTEC (1994) SAE, pp. 210.

2. Kullgren, Krafft, Tingvall, Lie (2005) “Combining Crash Recorders and Paired Comparison Technique: Injury Risk Functions in Frontal and Rear Impacts with Special Reference to Neck Injuries". National Highway Traffic Safety Administration, Washington DC, USA, pp. 404.

3. Minton, Murray, Stephenson, Gakasko (2002) "A Study of Lower Back Strain Injuries Resulting From Road Accidents", Transportation Research Laboratory, Wokingham, United Kingdom.
4. National Automotive Sampling System (2015) Department of Transportation, USA.

5. Farmer Wells, Werner (1999) "Relationship of Head Restraints Positioning to Driver Neck Injury in Rear End Crashes", Insurance Institute for Highway Safety, 31(6): 719-728.

6. Jakobsson, Norin, Bunketorp (2002) "In-Depth Study of Whiplash Associated Disorders in Frontal Impacts: Influencing Factors and Associated Consequences", Proceedings of the International Conference on the Biomechanics of Impacts, pp. 1-12.

7. Elbel, Kramer, Huber-Lang, Hartwig, Dehner (2009) "Deceleration During 'Real Life' Motor Vehicle Collisions - Predictors for the Risk of Sustaining a Cervical Spine Injury?" Patient Safety in Surgery 3(1): 5.

8. Smith, Smith (2007) The Lack of Correlation between Spinal Injuries and Change in Velocity in Rear Impacts-An Evaluation of Spinal Strain Proceedings of the 2007. International Whiplash Trauma Congress, USA.

9. Gabler, Fitzharris, Scully, Fildes, Digges, et al. Far Side Impact Injury Risk for Belted Occupants in Australia and the United States, USA, pp. 5-420.

10. Smith J (1999) An Analysis of 72 Real World Rear Impacts-An Initial Investigation Into Injury Factors, Warrendale PA, USA, p. 10.

11. Freeman, Michael (2008) Graph provided in email.

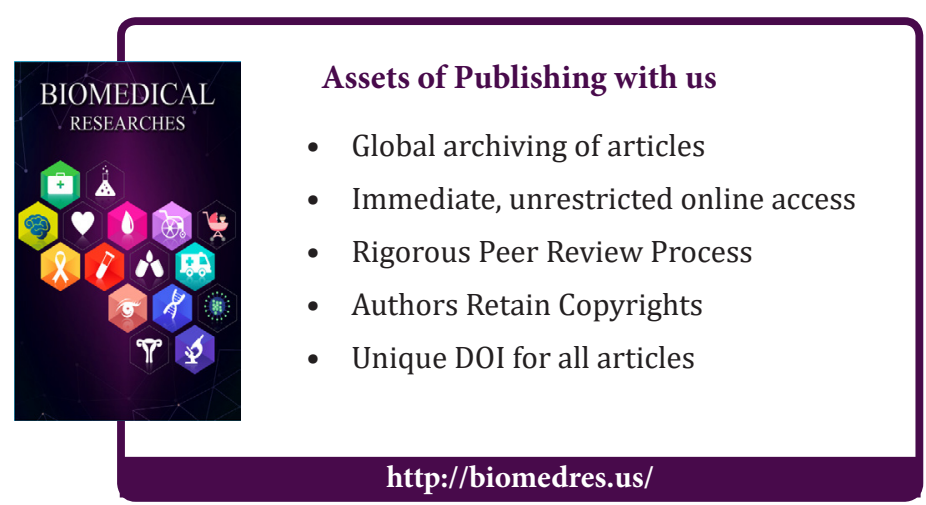

